\title{
Characterization of HIV-1 gag and nef in Cameroon: further evidence of extreme diversity at the origin of the HIV-1 group M epidemic
}

\author{
Marcel Tongo ${ }^{1,2}$, Darren P Martin ${ }^{3^{*}}$, Lycias Zembe ${ }^{1}$, Eitel Mpoudi-Ngole ${ }^{2}$, Carolyn Williamson ${ }^{1,4}$
} and Wendy A Burgers ${ }^{1 *}$

\begin{abstract}
Background: Cameroon, in west central Africa, has an extraordinary degree of HIV diversity, presenting a major challenge for the development of an effective HIV vaccine. Given the continuing need to closely monitor the emergence of new HIV variants in the country, we analyzed HIV-1 genetic diversity in 59 plasma samples from HIV-infected Cameroonian blood donors. Full length HIV gag and nef sequences were generated and phylogenetic analyses were performed.

Findings: All gag and nef sequences clustered within HIV-1M. Circulating recombinant form CRF02_AG predominated, accounting for $50 \%$ of the studied infections, followed by clade G (11\%), clade D and CRF37_cpx (4\% each), and clades A, F, CRF01_AE and CRF36_cpx (2\% each). In addition, 22\% of the studied viruses apparently had nef and gag genes from viruses belonging to different clades, with the majority (8/10) having either a nef or gag gene derived from CRF02_AG. Interestingly, five gag sequences (10\%) and three (5\%) nef sequences were neither obviously recombinant nor easily classifiable into any of the known HIV-1M clades.

Conclusion: This suggests the widespread existence of highly divergent HIV lineages in Cameroon. While the genetic complexity of the Cameroonian HIV-1 epidemic has potentially serious implications for the design of biomedical interventions, detailed analyses of divergent Cameroonian HIV-1M lineages could be crucial for dissecting the earliest evolutionary steps in the emergence of HIV-1M.
\end{abstract}

Keywords: HIV-1 diversity, West central Africa, RDP3, Maximum likelihood, PHYML

\section{Findings}

The Congo basin in west central Africa is thought to be the origin of HIV, where several cross-species transmission events from chimpanzees to humans occurred [1,2]. Cameroon, located in this region, has one of the most genetically diverse HIV epidemics in the world [3-6]. Alongside CRF02_AG, which accounts for more than half of infections in the country, circulating virus lineages include every known HIV-1M subtype, numerous circulating recombinant forms (CRFs) and a variety of apparently unique recombinant forms (URFs) [7]. The prevalence of HIV-1 in Cameroon is one of the highest

\footnotetext{
*Correspondence: Darrin.Martin@uct.ac.za; Wendy.Burgers@uct.ac.za

${ }^{3}$ Computational Biology Group, Institute of Infectious Diseases and Molecular Medicine, University of Cape Town, Cape Town, South Africa

'Division of Medical Virology, University of Cape Town, Cape Town, South Africa
}

in west central Africa, at 5.3\% [8]. This, together with the co-circulation of divergent variants of multiple clades, has created the conditions for frequent mixed infections and inter-clade recombination. Constantly improving phylogenetics-based analytical techniques and rapidly expanding HIV sequence datasets allow for better characterization of diverse sequences, and promise to yield important insights into the origin, evolution and spread of HIV-1.

Given the potential impact of HIV-1 diversity on both vaccine development and the sustainability of antiretroviral therapies, it is particularly important that molecular epidemiological surveillance is continued in HIV diversity hotspots such as Cameroon. In this study we have focused on characterizing the diversity of gag and nef genes of Cameroonian HIV-1 isolates. These genes are 
particularly relevant because they encode highly immunogenic proteins that are frequently included in candidate vaccines [9-11]. We sequenced 50 full length HIV-1 gag and 55 nef genes from 59 HIV-infected blood donors in Cameroon. To obtain a phylogenetic view of Cameroonian HIV diversity that explicitly accounted for the confounding effects of recombination, we performed extensive recombination-aware phylogenetic analyses of these new sequences along with publically available homologous HIV-1M gag and nef sequences from the Congo basin and a representative selection of the major known HIV lineages from the rest of the world. These representative sequences were selected to include the broadest diversity of sequences previously identified as belonging to these known clades by constructing maximum likelihood trees from all available gag and nef sequences for each clade, and selecting one sequence from each of the up to ten most basal lineages from the root of these clades.

Anonymously-donated HIV-infected blood units were collected between December 2006 and August 2007 from Yaoundé Central Hospital, Cameroon, in a study approved by the National Ethics Committee of the Cameroonian Ministry of Health and the University of Cape Town. Although no data on risk factors for HIV was available for the blood donors, they are believed to represent the general adult population of Yaoundé. All donors were antiretroviral therapy naïve and only age and gender information were obtained.

RNA was extracted from plasma samples, reverse transcribed and PCR amplified as described previously [12] using subtype non-specific HIV-1 primers for HIV-1 full length gag [12] and nef [13] genes, and sequenced. Sequenced fragments were assembled using ChromasPro. Full length gag and nef sequences were generated and aligned using MUSCLE with manual editing in MEGA5, together with a representative selection of 270 gag and 279 nef HIV sequences from the rest of the world and all other published gag (266) and nef (278) sequences from Cameroon and other west central African countries available in the LANL (http://www.hiv.lanl. gov/content/sequence/HIV/mainpage.html) and Genbank databases. Maximum likelihood phylogenetic trees were constructed from these sequences with 100 full maximum likelihood bootstrap replicates (implemented in PHYML [14]), following either complete removal of recombinant sequence fragments or the division of recombinant sequences into their constituent fragments by a blinded fully exploratory screen for recombination using RDP3 [15]. The recombination screen was fully exploratory in that every sequence was analysed for evidence of both intra- and inter-clade recombination. Either full nef and gag sequences or the sub-fragments of these sequences identified as having recombinant origins were classified as belonging to particular HIV clades if they clustered with reference sequences of these clades. Divergent sequences were defined as those residing on isolated branches outside of subtrees containing previously defined HIV-1 subtype or CRF lineages. Outlier sequences on the other hand were defined as those residing on basal branches of subtrees containing previously defined HIV-1 subtype or CRF lineages. Nucleotide sequences were deposited in GenBank [JX244899-JX244948 for gag and JX244949JX245003 for nef].

Clinical and demographic data of the HIV-infected Cameroonian study participants are summarized in Table 1. Individuals had a median age of 31 years and the majority were male. They had a median CD4 count of 432 cells $/ \mathrm{mm}^{3}$ and a median viral load of approximately 100000 RNA copies $/ \mathrm{ml}$.

All our sequences were derived from HIV-1 group $M$ viruses (Figure 1 and Additional files 1, 2). The sequences clustered with different clades and circulating recombinant forms distributed throughout the phylogenetic trees (Table 2), consistent with the breadth of HIV-1 diversity previously described in Cameroon. CRF02_AG-like viruses dominated the clade distribution, infecting $50 \%$ of the 46 participants for which both genes were sequenced (Figure 2). Participants infected with viruses having both nef and gag clustering within known HIV-1M clades included those belonging to clades G, D, A, and F. Subtype G sequences accounted for $11 \%$ of infections, subtype D for $4 \%$ and sub-subtypes A1 and F2 for $2 \%$ each. In addition to CRF02_AG, other CRFs identified were CRF37_cpx (4\%), and CRF01_AE and CRF36_cpx (2\% each). Additionally, in two samples for which only gag or nef was typed, these were classified as belonging to CRF11_cpx. Notably, despite subtypes B and C collectively accounting for approximately $75 \%$ infections worldwide [16], none of our sequences were classified as belonging to either of these clades.

In 10/46 samples from which both nef and gag sequences were analysed, they were classified as belonging to different clades from one another. One of the two gene sequences from 8/10 of these patients were classified as CRF02_AG (Figure 2 and Table 3). In addition, we detected numerous recombination breakpoints within the genome regions analysed. Among the newly sequenced gag genes, three (6\%) were identified as containing recombination breakpoints between gene segments that phylogenetically clustered

\section{Table 1 Characteristics of study individuals $(n=59)$}

\begin{tabular}{|c|c|}
\hline Age $^{a}$ (years) & $31(19-54)$ \\
\hline Sex (Male: Female) & 46: 13 \\
\hline CD4 count ${ }^{\mathrm{a}}$ (cells $/ \mathrm{mm}^{3}$ ) & $432(42-1972)$ \\
\hline Viral load ${ }^{a}$ (Log RNA copies/ml) & $4.93(3.29-6.32)$ \\
\hline
\end{tabular}

${ }^{a}$ Median and range. 


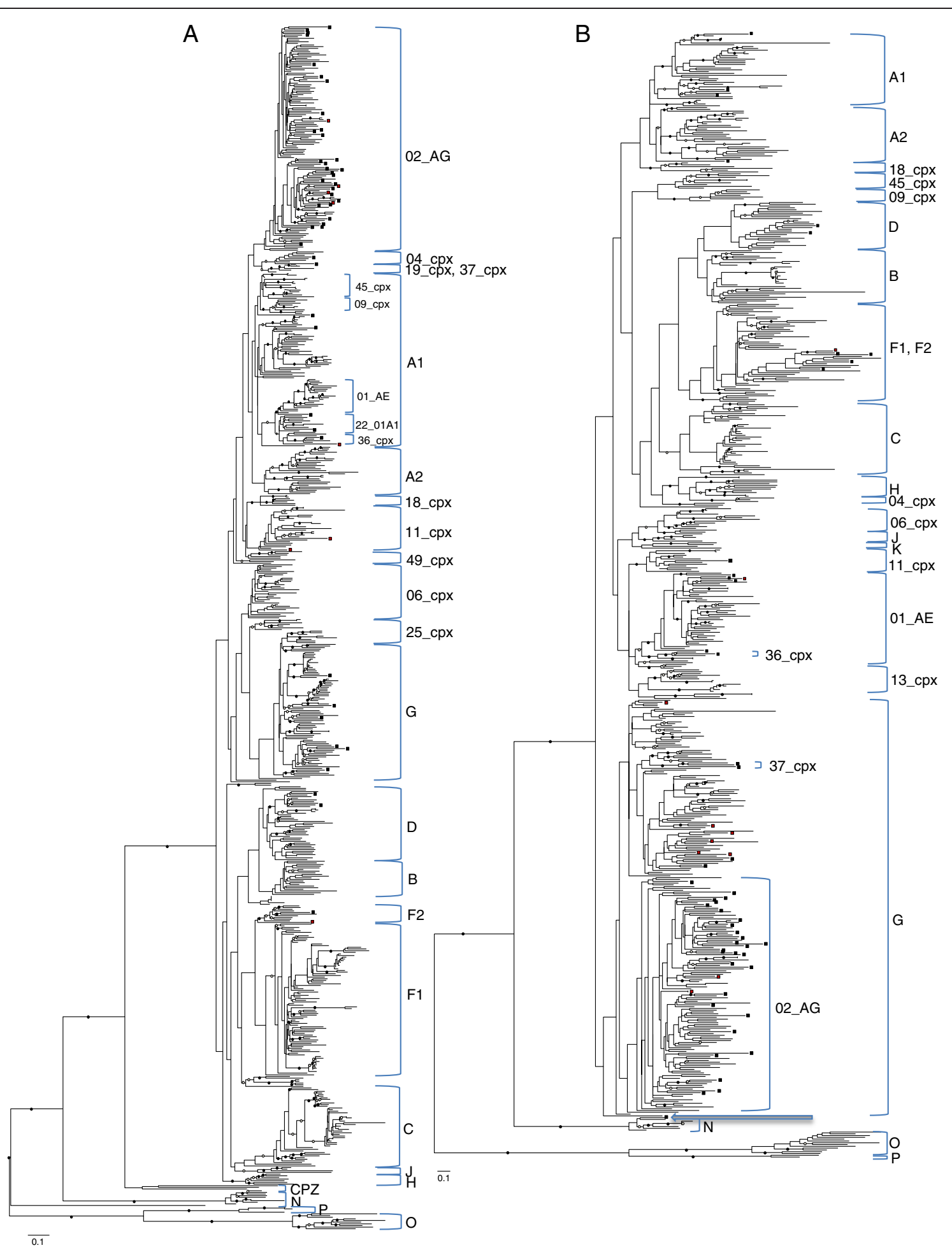

Figure 1 Maximum likelihood trees indicating the phylogenetic relationships between 727 gag (A) and 628 nef (B) sequences of HIV-1.

The trees were constructed from these sequences with 100 boostrap replicates following removal of recombinant sequence fragments by a blinded fully exploratory screen for recombination using RDP3. Black squares at the end of the branches represent the gag and nef sequences sampled from Cameroon in this study, while red squares represent intragene recombinant fragments in our samples. The gag tree was rooted using HIV-1 group N, O, P and SIV CPZ isolates, while the nef tree was rooted with HIV-1 group N, O and P isolates. Solid and open circles indicate branches with greater than $70 \%$ and $50 \%$ bootstrap support, respectively. The arrow in the nef tree indicates an outlier of both clades $\mathrm{G}$ and CRF02_AG. 
Table 2 Clade distribution of HIV-1 in Cameroon

\begin{tabular}{|c|c|c|c|}
\hline Sample ID & gag gene & nef gene & Genotype \\
\hline BSO1 & CRF02_AG & CRF02_AG & CRF02_AG \\
\hline $\mathrm{BSO} 2$ & A-like ${ }^{b}$ & CRF02_AG & URF \\
\hline $\mathrm{BSO3}$ & G & G & G \\
\hline BSO4 & G & $\mathrm{ND}^{c}$ & $G^{a}$ \\
\hline BSO5 & CRF02_AG & $\mathrm{ND}^{c}$ & CRF02_AG ${ }^{a}$ \\
\hline BS06 & CRF02_AG & CRF02_AG & CRF02_AG \\
\hline BS09 & CRF02_AG & $\mathrm{A} 1$ & URF \\
\hline BS10 & $\mathrm{A} 1$ & $\mathrm{~A} 1$ & $\mathrm{~A} 1$ \\
\hline BS11 & CRF02_AG & $\mathrm{F}$ & URF \\
\hline BS12 & G & G & G \\
\hline BS13 & CRF02_AG & $\mathrm{A} 1$ & URF \\
\hline BS14 & CRF02_AG & CRF02_AG & CRF02_AG \\
\hline BS16 & CRF02_AG & CRF02_AG & CRF02_AG \\
\hline BS18 & $N D^{c}$ & CRF02_AG & CRF02_AG ${ }^{\mathrm{a}}$ \\
\hline BS19 & CRF02_AG & CRF02_AG & CRF02_AG \\
\hline BS20 & $N D^{c}$ & CRF02_AG & CRF02_AG ${ }^{\mathrm{a}}$ \\
\hline BS21 & CRF02_AG & CRF02_AG & CRF02_AG \\
\hline BS22 & CRF02_AG & CRF02_AG & CRF02_AG \\
\hline BS23 & CRF02_AG & CRF02_AG & CRF02_AG \\
\hline BS24 & CRF37_cpx & CRF37_cpx & CRF37_cpx \\
\hline BS25 & CRF02_AG & $F$ & URF \\
\hline BS26 & CRF01_AE ${ }^{b}$ & CRF01_AE & CRF01_AE \\
\hline BS27 & CRF37_cp ${ }^{\mathrm{b}}$ & CRF37_cpx & CRF37_cpx \\
\hline BS29 & CRF02_AG & $U^{b}$ & URF \\
\hline BS30 & D & D & D \\
\hline BS31 & $N D^{c}$ & CRF02_AG & CRF02_AG ${ }^{\mathrm{a}}$ \\
\hline BS32 & CRF02_AG & CRF02_AG & CRF02_AG \\
\hline BS35 & $N D^{c}$ & CRF11_cpx & CRF11_cpx $x^{a}$ \\
\hline BS38 & CRF02_AG & CRF02_AG & CRF02_AG \\
\hline BS39 & CRF02_AG & CRF02_AG & CRF02_AG \\
\hline BS40 & CRF36_cpx & CRF36_cpx & CRF36_cpx \\
\hline BS42 & URF & CRF01_AE & URF \\
\hline BS43 & CRF02_AG & CRF02_AG & CRF02_AG \\
\hline BS44 & $N D^{c}$ & CRF02_AG & CRF02_AG ${ }^{\mathrm{a}}$ \\
\hline BS45 & CRF02_AG & CRF02_AG & CRF02_AG \\
\hline BS46 & G & G & G \\
\hline BS47 & CRF02_AG & CRF02_AG & CRF02_AG \\
\hline BS48 & G & G & G \\
\hline BS49 & F2 & $\mathrm{F}$ & F2 \\
\hline BS50 & CRF02_AG & CRF02_AG & CRF02_AG \\
\hline BS51 & G & G & G \\
\hline BS53 & CRF02_AG & CRF02_AG & CRF02_AG \\
\hline BS54 & D & D & D \\
\hline BS55 & CRF02 AG & $\mathrm{F}$ & URF \\
\hline
\end{tabular}

Table 2 Clade distribution of HIV-1 in Cameroon (Continued)

\begin{tabular}{|c|c|c|c|}
\hline BS56 & CRF02_AG & CRF02_AG & CRF02_AG \\
\hline BS57 & CRF11_cpx ${ }^{b}$ & $N D^{c}$ & CRF11_cp ${ }^{a}$ \\
\hline BS64 & CRF02_AG & CRF02_AG & CRF02_AG \\
\hline BS65 & CRF22_01A1 & CRF01_AE & URF \\
\hline BS66 & CRF02_AG & CRF02_AG & CRF02_AG \\
\hline BS71 & CRF02_AG & CRF02_AG & CRF02_AG \\
\hline BS72 & CRF36_cpx $/ F 2^{\mathrm{b}}$ & CRF01/F & URF \\
\hline BS73 & CRF02_AG & CRF02_AG & CRF02_AG \\
\hline BS74 & $N D^{c}$ & A-like ${ }^{b}$ & A-like ${ }^{a}$ \\
\hline BS75 & CRF02_AG & CRF02_AG & CRF02_AG \\
\hline BS77 & CRF02_AG & CRF02_AG & CRF02_AG \\
\hline BS78 & $N D^{c}$ & CRF01_AE & CRF01_AE ${ }^{a}$ \\
\hline BS79 & $N D^{c}$ & CRF02_AG & CRF02_AG ${ }^{a}$ \\
\hline BS81 & CRF02_AG & $\mathrm{ND}^{c}$ & CRF02_AG ${ }^{a}$ \\
\hline BS82 & $N D^{c}$ & CRF02_AG & CRF02_AG ${ }^{a}$ \\
\hline
\end{tabular}

within two distinct groups of the same clade, indicating that they were likely intra-clade recombinants. Whereas two of these (BS05 and BS55) were CRF02_AG/02_AG recombinants, one (BS57) was a CRF11_cpx/11_cpx recombinant (Table 3). Four of the newly sequenced nef genes (7\%) also showed evidence of intra-clade recombination, including three (BS12, BS48 and BS51) which were intra-subtype G recombinants and one (BS39) which was an intra-CRF02_AG recombinant. Whereas one of the newly sequenced gag genes (BS72) was apparently derived through recombination between F2 and CRF36_cpx parental viruses, one of the nef genes was apparently derived through recombination between F and CRF22_01A1 parental viruses.

The phylogenetic analysis of gag sequences derived from the Cameroonian samples further revealed four sequences (BS09, BS25, BS16 and BS42) situated on divergent branches near the base of the CRF02_AG subtree, highlighting the remarkable diversity within the CRF02_AG clade in Cameroon (indicated by blue squares in Additional file 1). Furthermore, five other Cameroonian gag sequences determined here branched very near the base of the clades that they grouped with: BS02 from the base of CRF09_cpx/CRF45_cpx, BS27 from the base of CRF37_cpx, BS26 from the base of CRF01_AE, BS57 from the base of the CRF11_cpx and BS72 from the base of the F2 and CRF36_cpx clades (blue arrows in Additional file 1). Similarly, in nef, three such sequences also branched from near the base of the clades that they were most closely clustered with: BS74 near the base of the A clade, BS42 near the base of the 


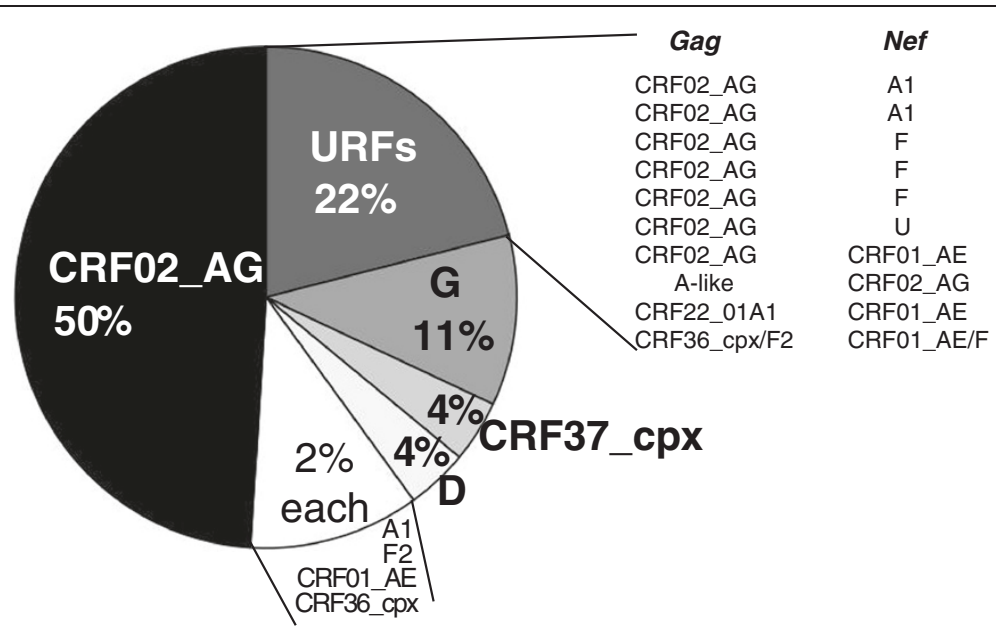

Figure 2 Pie chart summarizing the distribution of HIV-1 Group M clades and recombinant forms from full length gag and nef gene sequencing $(\mathbf{n}=\mathbf{4 6})$. Intergene recombinants are detailed in the right panel. The 13 samples that were typed in only one of the genes were excluded from this analysis.

CRF01_AE clade and BS29 near the base of the CRF02_AG/ G clades (blue arrows in Additional file 2).

Several studies have characterized HIV-1M sequences from Cameroon [3-7]. Our analysis of all available fulllength $g a g$ and $n e f$ gene sequences from west central Africa clearly reinforces the findings of these previous studies regarding the high degree of HIV-1M genetic diversity in this country. Also, unsurprising in the light of previous Cameroonian diversity studies, was our finding that most of the newly sampled sequences are likely CRF02_AG (accounting for 50\% of HIV-1M infections), with the other "pure" subtypes (G, D, A, and F) and CRFs (CRF11_cpx, 36_cpx, 37_cpx, and CRF01_AE) accounting for the remainder of infections.

CRF02_AG and clade G viruses are broadly distributed across west central Africa and have apparently been circulating stably there for many years [3,17-19], consistent with the presence of fragments of these viruses having been identified in a large number of CRFs and URFs that have been sampled from this region. Our analysis demonstrated that these two clades are highly diverse, and in most instances where gag and nef sequences from an individual patient had discordant clade classifications, the sequence of one of the genes clustered within the CRF02_AG clade, reinforcing the notion that this viral clade is a major contributor of genetic material to new recombinants [20]; an alternative explanation, however, could be that the gag and nef genes were amplified from different viruses co-infecting the same patients. Ongoing molecular and clinical surveillance will reveal whether new recombinants will begin to circulate stably, will harbor biological properties that favor their transmission, or will impact clinical outcomes.
Carr et al. [7] recently identified sequences that were outliers of various HIV-1M clades, and presented analyses that many of these viruses were likely URFs, which might explain the phylogenetic placement of these sequences on the outskirts of known clades. Although the majority of the outlier viruses found in our study were also URFs, they remained outliers after the removal of recombinant segments. It thus appears that these sequences represent viruses that are genuinely highly divergent and are possibly extant descendants of previously unknown early diverging

Table 3 Inter and intraclade recombinants

\begin{tabular}{|c|c|c|}
\hline Sample ID & gag gene & nef gene \\
\hline $\mathrm{BS} 02$ & A-like ${ }^{a}$ & CRF02_AG \\
\hline BS09 & CRF02_AG & A1 \\
\hline BS11 & CRF02_AG & $\mathrm{F}$ \\
\hline BS13 & CRF02_AG & $\mathrm{A} 1$ \\
\hline BS25 & CRF02_AG & $\mathrm{F}$ \\
\hline BS29 & CRF02_AG & $U^{a}$ \\
\hline BS42 & CRF02_AG & CRF01_AE ${ }^{a}$ \\
\hline BS39 & CRF02_AG & CRF02_AG/CRF02_AG \\
\hline BS55 & CRF02_AG/CRF02_AG & $\mathrm{F}$ \\
\hline BS05 & CRF02_AG/CRF02_AG & $N D^{b}$ \\
\hline BS65 & CRF22_01A1 & CRF01_AE \\
\hline BS72 & CRF36_cpx ${ }^{\mathrm{a}} / F 2^{\mathrm{a}}$ & CRF01_AE/F \\
\hline BS57 & CRF11_cpx ${ }^{\mathrm{a}} / \mathrm{CRF} 11$ _cpx & $N D^{b}$ \\
\hline BS12 & G & $\mathrm{G} / \mathrm{G}$ \\
\hline BS48 & G & $\mathrm{G} / \mathrm{G}$ \\
\hline BS51 & G & $\mathrm{G} / \mathrm{G}$ \\
\hline
\end{tabular}

${ }^{\mathrm{a} O u t l i e r ;}{ }^{\mathrm{b}} \mathrm{ND}$ : Not done. 
HIV-1M lineages. Such sequences could help tremendously with efforts to piece together the early evolutionary history of HIV-1M. For example, sequences such as BS29, which are outliers of both the CRF02_AG and G clades may help to resolve the controversy surrounding the origin of CRF02_AG [20-22].

In summary, our data show the predominance in an urban Cameroonian setting of HIV-1 CRF02_AG viruses alongside viruses belonging to known HIV-1M clades, URFs and currently unclassified divergent lineages. We are currently performing full-genome sequencing to further characterize the divergent sequences identified here.

\section{Additional files}

\section{Additional file 1: Detailed phylogenetic analysis of nucleotide} sequences in the gag gene. Maximum likelihood tree indicating the phylogenetic relationships between 727 gag sequences including all sequence identifiers. Blue arrows indicate the outlier sequences found in this study while the green arrows indicate the outlier sequences from previous Cameroonian sequences. Black squares at the end of the branches represent the gag sequences sampled from Cameroon in this study, while red squares represent intragene recombinant fragments in our samples. The blue squares show the new divergent branches formed by viruses sampled in this study. Sequence C.ZM.2006.ZM1464F appears to have been mis-labelled in the LANL database, and consistently groups with subtype A1.

Additional file 2: Detailed phylogenetic analysis of nucleotide sequences in the nef gene. Maximum likelihood tree indicating the phylogenetic relationships between 628 nef sequences including all sequence identifiers. Blue arrows indicate the outlier sequences found in this study while the green arrows indicate the outlier sequences from previously-characterized Cameroonian sequences. Black squares at the end of the branches represent the nef sequences sampled from Cameroon in this study, while red squares represent intragene recombinant fragments in our samples.

\section{Abbreviations}

HIV: Human Immunodeficiency Virus; CRF: Circulating recombinant form; URF: Unique recombinant form; RNA: Ribonucleic acid; PCR: Polymerase Chain Reaction.

\section{Competing interests}

The authors declare that they have no competing interests.

\section{Authors' contributions}

MT carried out the laboratory work and phylogenetic analyses, with assistance from LZ and DM. CW and EMN conceived of the study, and participated in its design and coordination. WB and DM supervised the work. MT, DM and WB wrote the manuscript. All authors read and approved the final manuscript.

\section{Acknowledgements}

The authors are grateful to Andile Nofemela and Roman Ntale for technical assistance with viral sequencing. This research was supported by the International Atomic Energy Agency (technical co-operation project RAF/6/ 029), Poliomyelitis Research Foundation (PRF) of South Africa and the University of Cape Town, for collaborative projects with partners in the global South. We thank Gerald Chege, Andreia Soares and Cobus Olivier for critical comments on the manuscript, and Mrs Kathryn Norman for administrative assistance. MT is a Carnegie Corporation PhD Fellow and received funding from the PRF. WAB is a Wellcome Trust Intermediate Fellow in Public Health and Tropical Medicine. The funders had no role in study design, data collection and analysis, decision to publish or preparation of the manuscript.

\section{Author details}

'Division of Medical Virology, University of Cape Town, Cape Town, South Africa. ${ }^{2}$ Institute of Medical Research and Study of Medicinal Plants, Yaoundé, Cameroon. ${ }^{3}$ Computational Biology Group, Institute of Infectious Diseases and Molecular Medicine, University of Cape Town, Cape Town, South Africa. ${ }^{4}$ National Health Laboratory Service, Groote Schuur Hospital, Cape Town, South Africa.

Received: 7 September 2012 Accepted: 14 January 2013 Published: 22 January 2013

\section{References}

1. Keele BF, Van Heuverswyn F, Li Y, Bailes E, Takehisa J, Santiago ML, BibolletRuche F, Chen Y, Wain LV, Liegeois F, Loul S, Ngole EM, Bienvenue Y, Delaporte E, Brookfield JF, Sharp PM, Shaw GM, Peeters M, Hahn BH: Chimpanzee reservoirs of pandemic and nonpandemic HIV-1. Science 2006, 313:523-526

2. Sharp PM, Hahn BH: Origins of HIV and the AIDS Pandemic. Cold Spring Harb Perspect Med 2011, 1:a006841.

3. Brennan CA, Bodelle P, Coffey R, Devare SG, Golden A, Harris B, Holzmayer V, Luk KC, Schochetman G, Swanson P, Yamaguchi J, Vallari A, Ndembi N, Ngansop C, Makamche F, Mbanya D, Gurtler LG, Zekeng L, Kaptue L, Hackett J Jr: The prevalence of diverse HIV-1 strains was stable in Cameroonian blood donors from 1996 to 2004. J Acquir Immune Defic Syndr 2008, 49:432-439.

4. Ragupathy V, Zhao J, Wood O, Tang S, Lee S, Nyambi P, Hewlett I: Identification of new, emerging HIV-1 unique recombinant forms and drug resistant viruses circulating in Cameroon. Virol J 2011, 8:185.

5. Machuca A, Tang S, Hu J, Lee S, Wood O, Vockley C, Vutukuri SG, Deshmukh $R$, Awazi $B$, Hewlett I: Increased genetic diversity and intersubtype recombinants of HIV-1 in blood donors from urban Cameroon. J Acquir Immune Defic Syndr 2007, 45:361-363.

6. Ndembi N, Abraha A, Pilch H, Ichimura H, Mbanya D, Kaptue L, Salata R, Arts EJ: Molecular characterization of human immunodeficiency virus type 1 (HIV-1) and HIV-2 in Yaounde, Cameroon: evidence of major drug resistance mutations in newly diagnosed patients infected with subtypes other than subtype B. J Clin Microbiol 2008, 46:177-184.

7. Carr JK, Wolfe ND, Torimiro JN, Tamoufe U, Mpoudi-Ngole E, Eyzaguirre L, Birx DL, McCutchan FE, Burke DS: HIV-1 recombinants with multiple parental strains in low-prevalence, remote regions of Cameroon: evolutionary relics? Retrovirology 2010, 7:39.

8. UNAIDS: AIDS Epidemic Update 2011. 2011. http://www.unaids.org

9. Masemola A, Mashishi T, Khoury G, Mohube P, Mokgotho P, Vardas E, Colvin M, Zijenah L, Katzenstein D, Musonda R, Allen S, Kumwenda N, Taha T, Gray G, McIntyre J, Karim SA, Sheppard HW, Gray CM, HIVNET 028 Study Team: Hierarchical targeting of subtype $C$ human immunodeficiency virus type 1 proteins by CD8+ T cells: correlation with viral load. J Virol 2004, 78:3233-3243

10. McMichael AJ, Haynes BF: Lessons learned from HIV-1 vaccine trials: new priorities and directions. Nat Immunol 2012, 13:423-427.

11 Stephenson KE, Li H, Walker BD, Michael NL, Barouch DH: Gag-Specific Cellular Immunity Determines In Vitro Viral Inhibition and In Vivo Virologic Control Following SIV Challenges of Vaccinated Rhesus Monkeys. J Virol 2012. Epub ahead of print.

12. Bredell H, Martin DP, Van Harmelen J, Varsani A, Sheppard HW, Donovan R, Gray CM, HIVNET028 Study Team, Williamson C: HIV type 1 subtype C gag and nef diversity in Southern Africa. AIDS Res Hum Retroviruses 2007, 23:477-481.

13. Artenstein AW, Hegerich PA, Beyrer C, Rungruengthanakit K, Michael NL, Natrapan C: Sequences and phylogenetic analysis of the nef gene from Thai subjects harboring subtype E HIV-1. AIDS Res Hum Retroviruses 1996, 12:557-560.

14. Guindon S, Lethiec F, Duroux P, Gascuel O: PHYML Online-a web server for fast maximum likelihood-based phylogenetic inference. Nucleic Acids Res 2005, 33:W557-W559.

15. Martin DP, Lemey P. Lott M, Moulton V Posada D, Lefeuvre P. RDP3: a flexible and fast computer program for analyzing recombination. Bioinformatics 2010, 26:2462-2463.

16. Hemelaar J, Gouws E, Ghys PD, Osmanov S, WHO-UNAIDS Network for HIV Isolation and Characterisation: Global trends in molecular epidemiology of HIV-1 during 2000-2007. AIDS 2011, 25:679-689. 
17. Montavon C, Vergne L, Bourgeois A, Mpoudi-Ngole E, Malonga-Mouellet G, Butel C, Toure-Kane C, Delaporte E, Peeters M: Identification of a new circulating recombinant form of HIV type 1, CRF11-cpx, involving subtypes A, G, J, and CRF01-AE, in Central Africa. AIDS Res Hum Retroviruses 2002, 18:231-236.

18. Hamel DJ, Sankale JL, Eisen G, Meloni ST, Mullins C, Gueye-Ndiaye A, Mboup S, Kanki PJ: Twenty years of prospective molecular epidemiology in Senegal: changes in HIV diversity. AIDS Res Hum Retroviruses 2007, 23:1189-1196

19. Faria N, Suchard MA, Abecasis A, Sousa JD, Ndembi N, Bonfim I, Camacho RJ, Vandamme AM, Lemey P: Phylodynamics of the HIV-1 CRF02_AG clade in Cameroon. Infect Genet Evol 2012, 12:453-460.

20. Zhang M, Foley B, Schultz AK, Macke JP, Bulla I, Stanke M, Morgenstern B, Korber $B$, Leitner $\mathrm{T}$ : The role of recombination in the emergence of a complex and dynamic HIV epidemic. Retrovirology 2010, 7:25.

21. Carr JK, Salminen MO, Albert J, Sanders-Buell E, Gotte D, Birx DL, McCutchan FE: Full genome sequences of human immunodeficiency virus type 1 subtypes $G$ and A/G intersubtype recombinants. Virology 1998, 247:22-31.

22. Abecasis $A B$, Lemey $P$, Vidal $N$, de Oliveira T, Peeters M, Camacho R, Shapiro B, Rambaut A, Vandamme AM: Recombination confounds the early evolutionary history of human immunodeficiency virus type 1: subtype $\mathrm{G}$ is a circulating recombinant form. J Virol 2007, 81:8543-8551.

doi:10.1186/1743-422X-10-29

Cite this article as: Tongo et al:: Characterization of HIV-1 gag and nef in Cameroon: further evidence of extreme diversity at the origin of the HIV-1 group M epidemic. Virology Journal 2013 10:29.

\section{Submit your next manuscript to BioMed Central and take full advantage of:}

- Convenient online submission

- Thorough peer review

- No space constraints or color figure charges

- Immediate publication on acceptance

- Inclusion in PubMed, CAS, Scopus and Google Scholar

- Research which is freely available for redistribution 